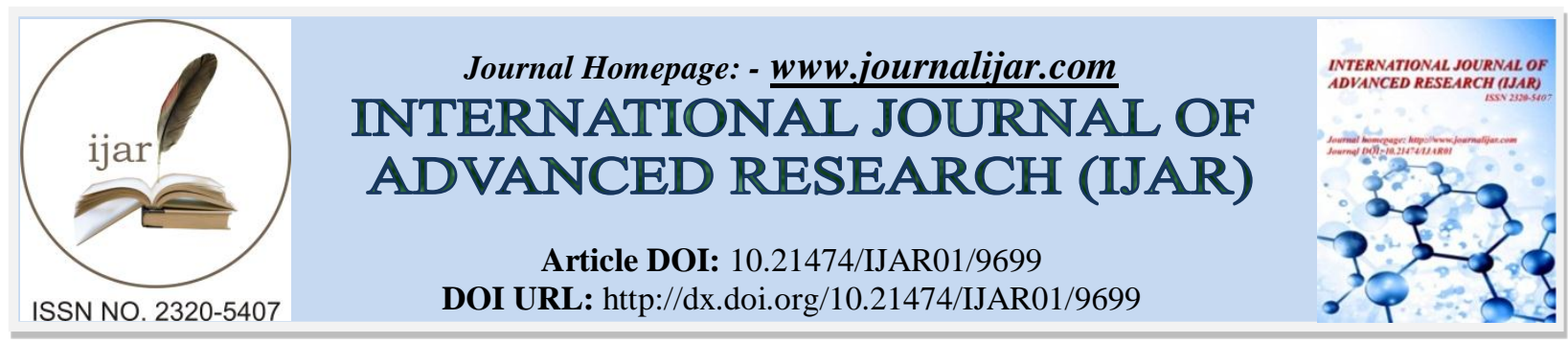

RESEARCH ARTICLE

\title{
EXTRACORPOREALSHOCKWAVE THERAPY FOR ACROMION PSEUDOATHROSIS A CASE REPORT.
}

Salman A. Almustafa.

MAOM, Dipl. AOM, BSPT Qatif Central Hospital at Ministry of Health Physical Therapy Department at Qatif city, Eastern P. KSA.

\section{Manuscript Info}

Manuscript History

Received: 08 July 2019

Final Accepted: 10 August 2019

Published: September 2019

Key words:-

Pseudoathrosis, $\quad$ Extracorporeal

shockwaves, Acromion.

\section{Abstract}

Fracture of Acromion is Uncommon because of its unique anatomical structure and location, usually it heals by itself with conservative therapies intervention and a subject's shoulder will get back to its functional performance without surgical approach; however, rare cases would not recover due to pseudoarthrosis is taking a place, surgical avoidance plus ongoing on noninvasive tools to manage acromion pseudoarthrosis with application of extracorporeal shockwave therapy medium to high intensity is my motivation to report this condition. Likewise; I have not encountered a published literature using extracorporeal shockwaves therapy for acromion specifically. A case of 36 old male active and alert has been referred to our physical therapy outpatient clinic for high density extracorporeal shockwaves therapy managing the acromion pseudoarthrosis after been going on ROM and strengthening rehabilitation program for 7 months. After having 6 sessions of ESWT, the acromion nonunion fracture got unified with complete patient condition recovery, the pain is abolished, ROM is full with restoration of functional abilities.

Copy Right, IJAR, 2019,. All rights reserved.

\section{Introduction:-}

The acromion is a large bony projection on the superior end of the scapula. Acromion fractures are rare injuries. They constitute $8 \%-16 \%$ of scapula fractures [1,2]. Recently, they are seen at the rate of $5 \%-6.9 \%$ as the complication of reverse shoulder arthroplasty [3]. Acromion fractures may occur as a result of shoulder trauma and overuse injuries. Acromion fractures may occur with glenoid process, scapula or clavicle distal fractures and disruption of superior shoulder suspensory complex [4]. There is no widely accepted treatment algorithm and fixation method of acromion fractures [5]. We aim to present a case report that contributes to diagnosis and treatment of acromion fracture that hasn't healed by itself applying noninvasive conservative treatment rather than surgery. Stimulation of bony union by means of various physical modalities has been widely used in clinical practice. Extracorporeal Shock Wave Therapy (ESWT) offers the most promise. It is based on the rationale that high speed sound causes lithotripsy and it was first used to crush kidney stones in 1980 (6). In 1986, Valchanov performed the first treatment of pseudoarthrosis with ESWT; his idea was to destroy the eburnated edges of the pseudoarthrosis and, at the same time, keep intact the periosteum. That way, by preserving the osteogenic tissue in the periosteum, rapid vascularization and consolidation of the bone were achieved (7). Clinical applications followed successfully like so experimental studies that showed osteoblast stimulation to osteogenesis (8, 9). We present our personal experience in the treatment of Acromion nonunion fracture with ESWT. The evolution of the

Corresponding Author:-Salman A. Almustafa.

Address:-MAOM, Dipl. AOM, BSPT Qatif Central Hospital at Ministry of Health Physical Therapy 569

Department at Qatif city, Eastern P. KSA. 
results obtained past 3 decades provide important data regarding indications, protocols of therapy, prognostic factors, percentages of the successes and future possible applications.

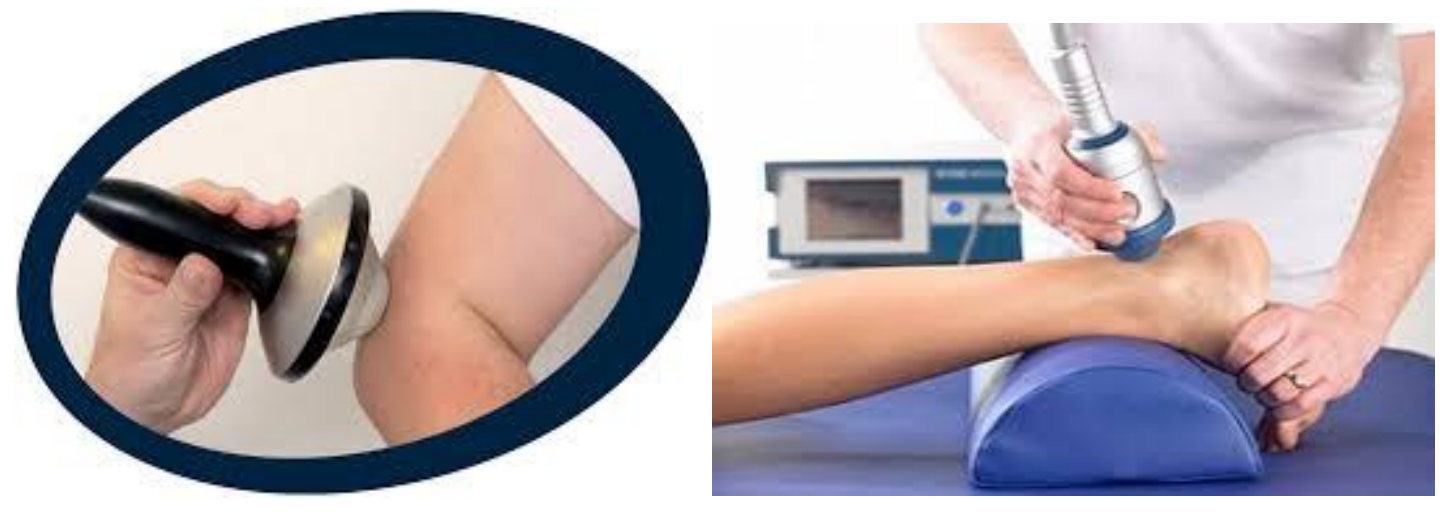

Case presentation:

A 36 years old male patient was driven to emergency unit in our hospital because of being hit by a vehicle when he was crossing the road while running. The patient was alert, oriented, and psychologically intact. He was not smoker without any medications dependency in that time. His family and he did not have any genetic or inherited diseases, he had no neurovascular deficits. Upon radiographic exam, he had left distal radius fracture with right acromion fracture that usually does not need any sort of fixation and heals by itself; the left arm completely had a cast with arm sling for 45 days. By the patient condition got stable, he was released from hospital to schedule a routine appointment in orthopedic outpatient clinic. On December, 2017 the patient referred to our physical therapy department after his cast been removed for left wrist ROM exercises and right shoulder ROM with shoulder girdle strengthening exercises. The patient was assigned with one of my colleagues for 6 visits, from his records, he was complaining of muscular spasmodic pain at both arm muscles with pain at left wrist fracture site. He was treated by ultrasound therapy on left wrist fracture to recall inflammatory mediators and fibroblast production to relieve pain and to enhance healing process. In the meantime, the patient underwent to left wrist and right shoulder ROM and strengthening exercises to boost patient upper limbs functional activates. After 3 weeks from last visit of physical therapy, the patient still had residual pain that is fixed on upper right shoulder as 8/10 VAS., In addition to feeling of right arm weakness with pain while he is lifting objects, the patient recalled that when his son was laying down on his right arm, the pain went worse on the same site of acromion site which referred down to the arm. On radiographic examination, the finding was nonunion acromion fracture (Figure 1.1). On July 23, 2018, the patient referred to our physical therapy department again for focused extracorporeal shockwaves therapy, the patient was complaining of pain, weakness and discomfort feeling at Rt. Shoulder and arm, the pain is as 8/10 VAS especially with lifting. Upon physical examination, the Rt. shoulder muscles power scored $(3+)$, the ROM is full with no neurovascular deficits, the only complaint is the pain at acromion site plus shoulder muscles weakness. According to (Morreti et al.,2009), "In the treatment of the pseudoarthrosis we used a range of energy flow density (EFD) between 0,22 and $1,10 \mathrm{~mJ} / \mathrm{mm} 2$ as tolerated and we applied 4000 pulses by an electromagnetic device (Minilith SL1-STORZ)", based on this and after taking clear verbal and written patient consent, the first visit ESWT parameters are $0.45 \mathrm{mj} / \mathrm{mm} 2$ which is high density energy (focused) and 1500 pulses with $4 \mathrm{~Hz}$ frequency. The patient released from clinic with instructions to not carry heavy weights and go over free active exercises of the shoulder. The second visit, I tended to raise the energy flow density to $0.55 \mathrm{mj} / \mathrm{mm} 2$ and pulses to 2200 to enhance more vascularization and consolidation at fracture site. The patient pain is going less on the second visit, it reduced from 7/10 VAS to 5/10 at acromion site, also patient was able to use his arm in his basis live activities without disabling pain. From third session to sixth, our parameters are $0.55 \mathrm{mj} / \mathrm{mm} 2$ with 2500 pulses and $3 \mathrm{~Hz}$ frequency, patient pain subsided to $2 / 10$ then $1 / 10$ VAS at the sixth session. Patient was significantly cured and released from that stressing pain. Patient had his own routine follow up appointment with his orthopedic physician who had ordered x-ray imaging in order to inspect how much the callus formation been built and how much the fracture gap was resolved, amazingly, the pseudoarthrosis is totally healed (Figure 2.1) with no gapping been recorded which ranked ESWT as number one intervention to treat delayed nonunion fractures and pseudoarthrosis. 


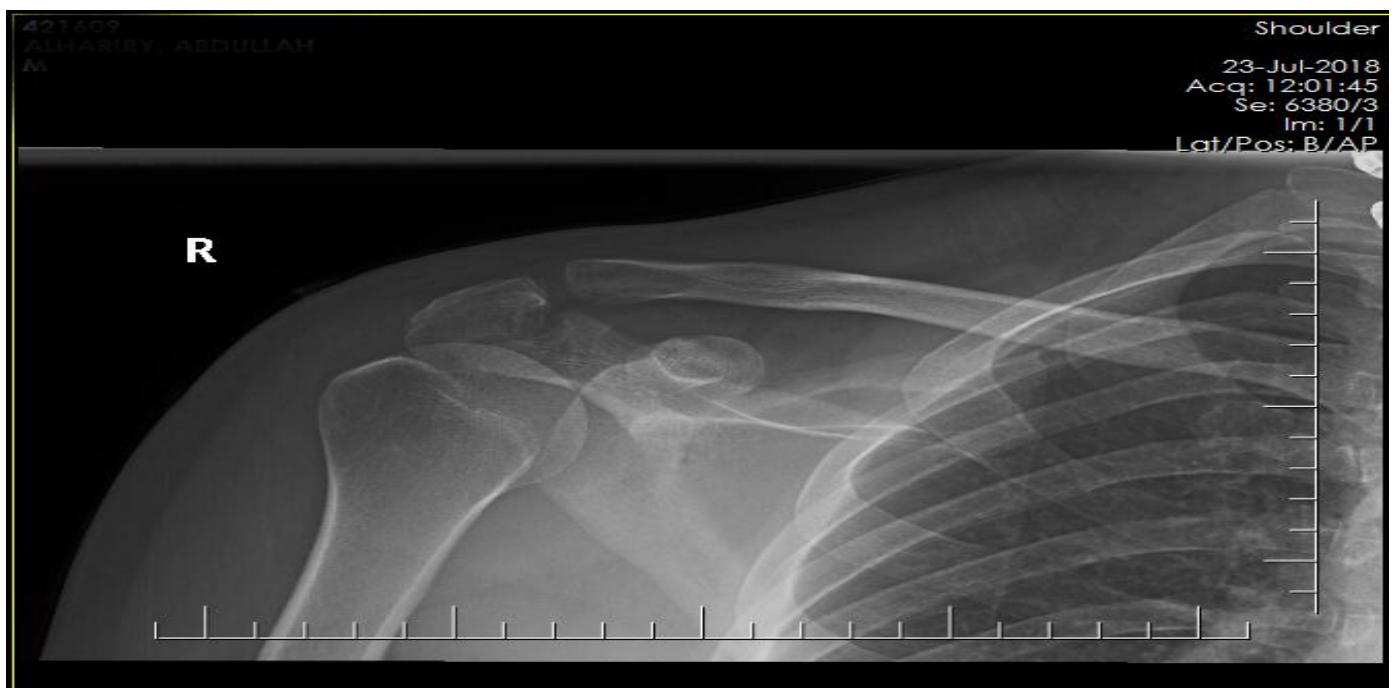

Figure 1.1:-



Figure 2.1:-

\section{Discussion:-}

Focused ESWT intervention is a number one treatment option to heal pseudoarthroisis, the case presentation has been successful progression outcomes applying ESWT to treat acromion pseudoarthrosis, a condition that usually heals spontaneously without any interventions, however; some cases might not due to mechanical and physiological limitations. According to the case presentation, the patient chronological referring is reasonable and evidence based supported, after engaging patient in ROM and strengthening exercises for about 2 months then discharge him to return back to his normal life activities, he came back with right shoulder pain at acromion site that has been nonunion for about 6 months since initial physical therapy visit, we underwent applying ESWT for 6 visits with great pseudoarthrosis healing and patient recovery outcomes, we aimed to activate vascularization and consolidation processes that enhance pseudoarthrosis unity, the pain subsided from $8 / 10$ to $0 / 10$, the functional performance of the patient's Rt. Shoulder went normally back without relapse or recurrence incidence after a routine follow up appointment within 2 months of discharge date, also there were no any complications been reported of using ESWT which ranks ESWT as best noninvasive approach managing nonunion pseudoarthrosis fractures. 


\section{Conclusion:-}

Focused ESWT is definitely first choice treatment to treat Acromion pseudoarthrosis to activate vascularization and consolidation enforcing fracture healing then returning back to active rehabilitation program which helping different patients resuming to their usual life style or sports activities.

\section{References:-}

1. T.P. Goss, The scapula: coracoid, acromial, and avulsion fractures, Am. J. Ortho.(Belle Mead N. J.) 25 (1996) $106-115$.

2. J.M. Lantry, C.S. Roberts, P.V. Giannoudis, Operative treatment of scapularfractures: a systematic review, Injury 39 (2008) 271-283.

3. L.A. Crosby, A. Hamilton, T. Twiss, Scapula fractures after reverse total shoulder arthroplasty: classification and treatment, Clin. Orthop. Relat. Res.469 (2011) 2544-2549.

4. B. Mulawka, A.R. Jacobson, L.K. Schroder, P.A. Cole, Triple and quadruple disruptions of the superior shoulder suspensory complex, J. Orthop. Trauma 29 (June (6)) (2015) 264-270.

5. B.W. Hill, J. Anavian, A.R. Jacobson, P.A. Cole, Surgical management of isolated acromion fractures: technical tricks and clinical experience, J. Orthop. Trauma28 (May (5)) (2014) e107-e113

6. Chaussy C, Brendel W, Schmiedt E. Extracorporeally induced destruction of kidney stones by shock waves. Lancet. 1980 Dec 13;2(8207):1265-8.

7. Valchanov VD, Michailov P. High energy shock waves in the treatmentof delayed and nonunions of fractures. Int Orthop. 1991;15:181-4.

8. Martini L, Giavaresi G, Fini M, et al. Early effects of extracorporeal shock wave treatment on osteoblast-like cells: a comparative study between electromagnetic and electrohydraulic devices. J Trauma. 2006 Nov;61(5):1198-206.

9. Moretti B, Notarnicola A, Tamma R, et al. Osteoblast repair action induced by ESWT. 11th Congress ISMST, Juan Les Pins, Antibes, France, 5-7 June 2008. Abstracts-book: 42-43. 